\title{
VOLUME-LIMITED SPECTROSCOPIC BINARY STATISTICS
}

\author{
J. Fisher, ${ }^{1}$ K.-P. Schröder, ${ }^{1}$ and Robert Connon Smith ${ }^{1}$
}

RESUMEN

Derivamos la distribución de períodos $(P)$, masas de las primarias $\left(m_{1}\right)$ y cocientes de masa $(q)$ para la población local de binarias de campo estudiando una muestra de 371 binarias espectroscópicas limitada por volumen, con $d \leq 100 \mathrm{pc} \mathrm{y} M_{v} \leq 4$. La muestra fue obtenida utilizando el catálogo de Batten, datos de R.F. Griffin y el catálogo HIPPARCOS. Las SB2 se usan para calibrar un tratamiento tipo Monte Carlo para la distribución de las $q$ de las SB1, lo cual da una distribución de $q$ total con un pico en $q \approx 1$. También se evalúa la completez y los sesgos específicos de los parámetros. Se encuentra un número apreciable de sistemas con períodos intermedios a largos, lo cual puede ser importante para la distribución de masas de las enanas blancas.

\section{ABSTRACT}

We derive the period $(P)$, primary mass $\left(m_{1}\right)$ and mass ratio $(q)$ distributions of the local population of field binaries by studying a volume-limited sample of 371 spectroscopic binaries (SBs) in the solar neighbourhood $d \leq 100 \mathrm{pc}$ and $M_{v} \leq 4$. The sample was collated using the Batten catalogue, data of R.F. Griffin and the HIPPARCOS catalogue. The SB2s are used to calibrate a Monte-Carlo approach to the $q$ distribution of SB1s, giving a total $q$ distribution confirming a peak at $q \approx 1$. Completenesses and parameter-specific biases are also assessed. A substantial number of systems with intermediate to long periods are found which may have significant consequences for the mass-distribution of WDs.

Key Words: BINARIES: SPECTROSCOPIC - STARS: STATISTICS

\section{PERIOD $(P)$ DISTRIBUTION}

The periods were found directly from the SB data $^{2}$ and divided into categories as given in Figure 1 ,

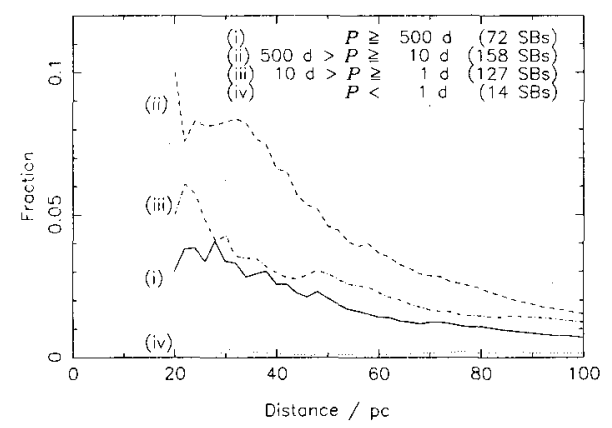

Fig. 1. SB Period distributions $\left(d \leq 100 \mathrm{pc}\right.$ and $\left.M_{v} \leq 4\right)$.

which shows the behaviour of the period distributions for different volumes within our sample. We are clearly missing a large fraction of the systems but can extrapolate using the curves from $100 \mathrm{pc}$ to around $40 \mathrm{pc}$ (where the data become unreliable due to low numbers) to determine what the fractions would be at $0 \mathrm{pc}$ (the fractions of binary/multiple systems to

\footnotetext{
${ }^{1}$ Astronomy Centre, University of Sussex, Falmer, Brighton, BN1 9QJ, UK (jfisher@pact.cpes.susx.ac.uk).

${ }^{2}$ We wish to express our special gratitude to R.F. Griffin for generously allowing use of his unpublished data.
}

all star systems). The extrapolations give the following estimates of the fractions: $0.087,0.255,0.107$ and 0.0015 for categories (i) to (iv) respectively, giving a total fraction of $0.45 \pm 0.05$. Although the data are for SBs, the extrapolations will be the fractions of all binary/multiples since all binaries would be detectable as SBs at $0 \mathrm{pc}$.

\section{2. $M_{1}$ AND $Q$ DISTRIBUTIONS}

The primary masses (distribution not shown) were estimated from $M-L$ relationships derived using the evolution code of Peter Eggleton (Pols et al. 1998), according to a system's location on an HRD (the HIPPARCOS parallax being used to find $M_{v}$, to which an offset was added to give the primary $M_{v}$ ).

The SB2 $q\left(=m_{2} / m_{1}\right)$ distribution was found directly from the observed $K_{1}$ and $K_{2}$ orbital semiamplitudes. For SB1s however the nearest one can get directly is the following function of $q$, using the mass function, $f(m)$, and $m_{1}: f(m) / m_{1}=$ $q^{3} \sin ^{3} i /(1+q)^{2}$. To find the SB1 $q$ distribution a Monte-Carlo method was then used. A number of assumptions have always had to be made in the past for the method to work, but by using the HIPPAR$C O S$ data we have been able to introduce a number of refinements. (For other work on $q$ distributions 
see, for example, Halbwachs et al. 2003, Boffin, Cerf \& Paulus 1993, Hogeveen 1991 and Trimble 1990.)

By use of the above $m_{1}$ 's, the dual problems of having to make assumptions about the unknown masses and of being restricted to stars of a particular luminosity class could be avoided. We also used random inclinations, $i, \alpha_{0}<i \leq 90^{\circ}$, where $\alpha_{0}$ is a minimum cutoff angle (systems with $i$ near $0^{\circ}$ being difficult to detect as SBs) to avoid having to assume an average value of $\sin ^{3} i$ on the one hand, or a relationship for the probability of detecting a system with inclination $i$ on the other. The SB2s were used to calibrate $\alpha_{0}$ for all SBs (assuming SB2s and SB1s have the same selection criteria - see $\S 3$ ) by comparing the $f(m) / m_{1}$ distribution for SB2s from two different sources: (i) directly from their $q$ values and random $i$ 's, for different $\alpha_{0}$, and (ii) indirectly from $f(m)$ and $m_{1}$. By this method the best match was found to be for $\alpha_{0}=24^{\circ} \pm 0.5^{\circ}$.

To perform the Monte-Carlo simulation a variety of plausible $q$ distributions were considered and the resultant $f(m) / m_{1}$ distributions calculated for random $i$ 's. From a variety of exponential-like, 'hump' and step functions the best (and least arbitrary) $q$ distribution was found to be the step function given in Figure 2, shown combined with the SB2 $q$ distribution (justifiable as the SB2 and SB1 distributions are independent of each other). The effects of adding the next best SB1 $q$ distributions are also shown. The figure clearly shows a peak towards $q=1$. Further, this peak comes from the SB2 contribution, derived directly from the observed data, and so is unaffected by uncertainties in the SB1 distribution.

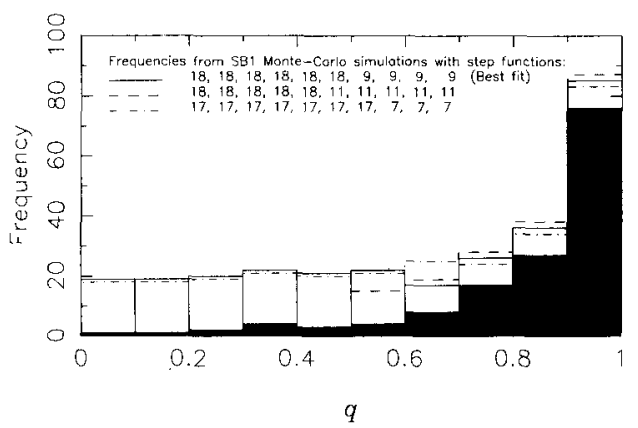

Fig. 2. $q$ distribution for all SBs, SB2s on the bottom (filled) and SB1s above (unfilled).

\section{DISCUSSION}

The fractions of SB2s and SB1s within $100 \mathrm{pc}$ behave very similarly for different limiting $M_{v}$ (figure not shown), to some degree justifying using parameters derived from SB2s, such as the value of $\alpha_{0}$, for
SB1s too. However, there is another possible selection effect acting upon the $m_{1}$ distribution: the possibility of a lower detection rate for less luminous binaries. This would be reflected in a less pronounced increase in the observed $m_{1}$ distribution towards smaller masses, as compared to the true present-day mass function (PDMF). In the observed distribution, $d N / d \log m_{1} \propto m_{1}^{-2.8}$, while for single stars it is approximately $\propto m^{-4.8}$. However, we need to know if this difference is genuine or due to a selection effect (or both). To see this we look at the variation in detected SB fraction with volume and compare it with the variation with $M_{v}$ (figures not shown). The former shows a decrease by a factor of $\sim 5$ from $25-100 \mathrm{pc}$, the latter by a factor of $\sim 4.3$ over a corresponding range of $M_{v}=1$ to 4 . The latter decrease could again be due to a shallower PDMF or to a selection effect, but the former could be due only to the increasing incompleteness with increasing volume (and decreasing $m_{v}$ ). The fact that the two fractions fall off by approximately the same factor shows that the decrease with $M_{v}$, and hence the shallower PDMF, are due to a selection effect. Consequently, the (corrected) PDMF and IMF of binary primaries are nearly identical to those of single field stars in the solar neighbourhood (Schröder \& Sedlmayr 2001).

Finally, the large fraction of binaries (26\% in a complete sample) in category (ii), which would interact only when the primary has evolved onto the RGB or AGB, are of interest with respect to the WD population. In general, the primaries of these systems would leave a WD similar to the end product of the (undisturbed) evolution of a comparable single star, but with a mass a bit smaller: $m_{\text {WD }} \approx 0.4-0.55 \mathrm{M}_{\odot}$. These WDs may therefore be responsible for a hidden shift in the peak of the WD mass distribution from around $0.61 \mathrm{M}_{\odot}$ (as predicted by most single star population synthesis models) to around, or just under, $0.55 \mathrm{M}_{\odot}$ (binarity being difficult to detect in double-degenerate systems).

\section{REFERENCES}

Boffin, H. M. J., Cerf, N., \& Paulus, G. 1993, A\&A, 271, 125

Halbwachs, J.-L., Mayor, M., Udry, S., \& Arenou, F. 2003, A\&A, 397, 159

Hogeveen, S. J. 1991, PhD thesis, Univ. of Illinois

Pols, O., Schröder, K.-P., Hurley, J., Tout, C. A., \& Eggleton, P. 1998, MNRAS, 298, 525

Trimble, V. 1990, MNRAS, 242, 79

Schröder, K.-P. \& Sedlmayr, E. 2001, A\&A, 366, 913 